\title{
Illumination change at a depth edge can reduce lightness constancy
}

\author{
JAMES A. SCHIRILLO \\ University of San Francisco, San Francisco, California \\ and \\ LAWRENCE E. AREND \\ David Sarnoff Research Center, Princeton, New Jersey
}

\begin{abstract}
Lightness constancy requires that a surface retain its lightness not only when the illumination is changed but also when the surface is moved from one background to another. Occlusion of one surface by another frequently results in a retinal juxtaposition of patches under different illuminations. At such edges, retinal luminance ratios can be much higher than in scenes with a single illumination. We demonstrate that such retinal adjacencies can produce failures of lightness constancy. We argue that they are responsible for departures from perfect lightness constancy in two prior experiments that examined the effects of depth relations on lightness constancy.
\end{abstract}

There are at least three different environmental challenges to perceiving a given surface material as having constant achromatic surface color-that is, lightness constancy. ${ }^{1}$

Change in illumination. The problem that has historically received the most attention is that of maintaining the appearance of constant achromatic surface color as the illumination on a surface is changed. Previous experiments have shown that excellent lightness constancy can occur when multiple illuminations fall on surface patches in a single depth plane (Arend \& Goldstein, 1987a, 1987b, 1990).

Change in shape. Surfaces of curved objects must also be perceived as having constant lightness as the surfaces vary in 3-D orientation and depth. Under some circumstances, this can be treated as a special case of the illumination invariance problem (Mingolla \& Arend, 1993; Todd \& Mingolla, 1983). The degree of lightness constancy with 3-D shading varies from very good to poor, depending on the distribution of reflectances over the surface (Mingolla \& Arend, 1993).

Change in background. A surface in a scene with a single illumination should retain its lightness as it is moved from one background to another. In Arend and Goldstein's (1987a, 1987b, 1990) experiments, the test

This research was conducted at the Schepens Eye Research Institute, Harvard Medical School, and the Vision Research Laboratory, University of Chicago. It was supported by Grants AFOSR-89-0377 to L.E.A., T32 EY-07098 to J.A.S., and EY-07390 to S. Shevell. We thank $\mathrm{R}$. Goldstein and Linda Glennie for writing the experimental programs and A. L. Gilchrist for providing additional background information and useful comments. Correspondence should be addressed to J. A. Schirillo, Department of Psychology, University of San Francisco, 2130 Fulton Street, San Francisco, CA 94117-1080 (e-mail: schirilloj@) usfea.edu). and standard patches were surrounded by identical reflectances and were well removed from the illumination edge. Recent experiments by Whittle (1992), Arend and Spehar (1993), and Arend and Arend (1994) have shown that moving a gray patch from a black background to a white background produces small lightness changes. The latter two experiments also showed that the sizes of the errors due to background changes were independent of illumination variation. That is, changing the background from a black reflectance to white caused a fixed, small $(<1.5$ Munsell value steps) deviation from the lightness required for illumination-invariance lightness constancy. The small size of the constancy failure when a black background is substituted for a white is likely due to the limited dynamic range of Lambertian reflectance, typically less than 50:1 (Eastman Kodak, 1986). It is well known that varying the luminance of a surrounding annulus over several $\log$ units dramatically alters the brightness of a test patch (Heinemann, 1955).

In the viewing of some natural scenes, luminance ratios much larger than 50:1 can arise between adjacent patches in the retinal images. The occlusion of one surface by another frequently results in the retinal juxtaposition of patches under different illuminations. Since different depth planes may have very different illuminations (e.g., over 100 to 1; see Jacobsen \& Gilchrist, 1988; McCann, 1987), retinally adjacent objects whose physical depths are different may have a luminance ratio that significantly exceeds the maximum ratio among reflectances under the same illumination.

Gilchrist (1977) noted the frequent occurrence of illumination changes at depth edges and proposed a "coplanar ratio hypothesis." It states that "perceived lightness might be determined primarily by ratios within perceived planes rather than by all retinal ratios regardless of perceived depth" (p. 186). Models that construct 
lightness distributions by integration of luminance ratios at edges (Arend \& Goldstein, 1987a; Gilchrist, Delman, \& Jacobsen, 1983; Land \& McCann, 1971) produce correct relative lightnesses only if no illumination edges are included.

To test the coplanar ratio hypothesis, Gilchrist (1977) placed two gray papers against the far wall of a brightly illuminated room, and another one on the wall of a closer, dimly illuminated room. He then placed a test patch in the doorway between the two rooms. By varying the apparent interposition of patches, he made the test patch appear to lie in the same depth plane as did either the far papers or the near papers. This created the appearance of a test patch separated in apparent depth from some surfaces, while remaining retinally adjacent to them. The luminance ratios among a black paper in the near room, the test patch, and a white paper in the far room were $0.033: 1: 72$, respectively, a much larger total range $(\approx 1: 2000)$ than that spanned by black and white surfaces under a single illuminant.

In each condition, Gilchrist's observers chose from a separately illuminated comparison chart a patch that matched the test patch. The comparison chart consisted of gray Munsell papers ranging from Value 2.0/ to 9.5/ in steps of $0.5 /$ (a luminance range of 1:30).

Gilchrist's (1977) results were in qualitative agreement with his predictions. When the test patch appeared in the same depth plane as did the black patch in the near room (30:1 reflectance ratio between the two patches), the observers matched the test patch with a white Munsell chip $(V=9.0 /)$. When the same test patch was seen as coplanar with the white patch in the far room (1:72 reflectance ratio between the two patches) the observers matched the test patch with a comparison patch of Munsell value $\mathrm{V}=3.5 /$, a dark gray.

Although the lightness difference of 5.5 Munsell steps from the depth manipulation is dramatic, it falls short of the 30:1 and 72:1 ratios (7.5 Munsell steps) predicted under the coplanar ratio hypothesis for perfect performance in the near and far rooms, respectively. For Gilchrist's (1977) observers to have shown complete constancy, they would have needed to select the blackest comparison patch available $(\mathrm{V}=2.0 /)$ when the test was coplanar with the far white patch, and the whitest comparison patch available $(V=9.5 /)$ when the test was coplanar with the near black patch. Converting the Munsell values that Gilchrist obtained to reflectances gives a luminance ratio of 6.6:1. In a CRT replication of Gilchrist's study, with both test and coplanar patch ratios restricted to 30:1, Schirillo, Reeves, and Arend (1990) obtained a ratio match of $2.7: 1$.

In this paper, we examine the possibility that Gilchrist (1977) and Schirillo et al.'s (1990) failure to obtain complete constancy was due to the high ratio of adjacent retinal illuminations, which could influence the lightness of the test patch through local contrast. Our strategy was to compare lightness matches for two patterns. In one, we conceptually replicated the conditions of
Schirillo et al. (1990) and Gilchrist (1977), by placing the illumination edge immediately adjacent to the standard patch. In the second condition, we moved the illumination edge far enough away from the standard patch to eliminate local contrast between the standard patch and patches in the other illumination. We found results similar to those of Schirillo et al. (1990) in the first condition, and nearly perfect lightness constancy in the second, thus supporting our hypothesis.

\section{METHOD}

\section{Equipment}

The patterns shown to three of the observers (J.S., D.A., and L.A.) were presented on a carefully calibrated Tektronix 690SR high-resolution color monitor under control of an Adage 3000 image processor and a VAX 11/750 minicomputer. ${ }^{2}$ Three additional observers (L.C., S.G., and I.B.) were shown identical luminance patterns on a carefully calibrated Sony 17 -in. color monitor under control of a Pixar II Image Processor and a Sun 3/150 Workstation. ${ }^{3}$ To check for consistency between the two physical display systems, Observer J.S. was run on both machines, with comparable results. Observers controlled the luminance of the test patch by moving a hand-held cursor horizontally over a high-resolution graphics tablet.

\section{Stimuli}

The stimuli (Figure 1) were simulations of arrays of uniformly illuminated, achromatic $(6500 \mathrm{~K})$, matte papers lying in two stereoscopically defined depth planes. At the beginning of each session, the observer adjusted a mirror stereoscope to provide stable, comfortable fusion of the right- and left-eye images. Angular disparities between the right- and left-eye images were calculated to place the far stimulus plane $30 \%$ farther away than the near, verified by observers' estimates.

The plane that appeared closer to the observer (subsequently called the near Mondrian) was always presented on the bottom portion of the screen (Figure 1) and subtended $20^{\circ} \times 21^{\circ}$ of visual angle. The near Mondrian included both the $2.8^{\circ}$ square test patch, centered in an $8.3^{\circ}$ square "annulus" (lower patch), and the $2.8^{\circ}$ square comparison patch, surrounded by an $8.3^{\circ}$ square annulus (upper patch). The comparison patch was placed near the illumination (and depth) edge. In the entire-annulus condition, the entire annulus surrounded the comparison patch in the near plane (Figure 1a). In the split-annulus condition, the top half of the annulus lay in the far plane, making the comparison patch lie directly adjacent to the illumination edge (Figure $1 \mathrm{~b}$ ).

The plane that appeared farther from the observer (subsequently called the far Mondrian) was always presented on the top portion of the screen and subtended $20^{\circ} \times 7^{\circ}$.

The simulated reflectances of all patches except the test patch were fixed throughout the experiment. Luminances of the individual rectangular patches varied from 0.04 to $75 \mathrm{~cd} / \mathrm{m}^{2}$. The two depth planes had different simulated illuminations. The illumination on the upper half of the comparison annulus was that of the depth plane in which it was perceived to lie.

The phosphor triads were invisible at the 45 -in. viewing distance. Only the monitor screen was illuminated in an otherwise dark room. The resulting display was a convincing simulation of an array of reflective surfaces with a depth edge separating two regions illuminated at different levels by unseen sources.

\section{Subjects}

Six observers participated, the 2 authors and 4 volunteers. All 6 had prior experience in making lightness matches. The 4 naive vol- 


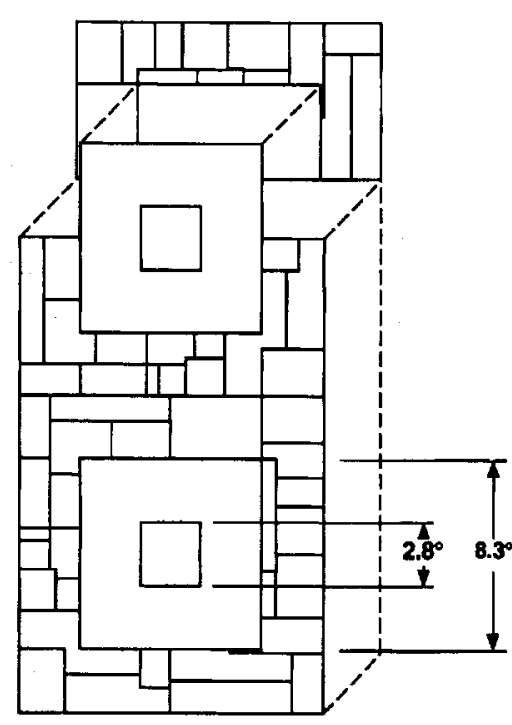

(a)

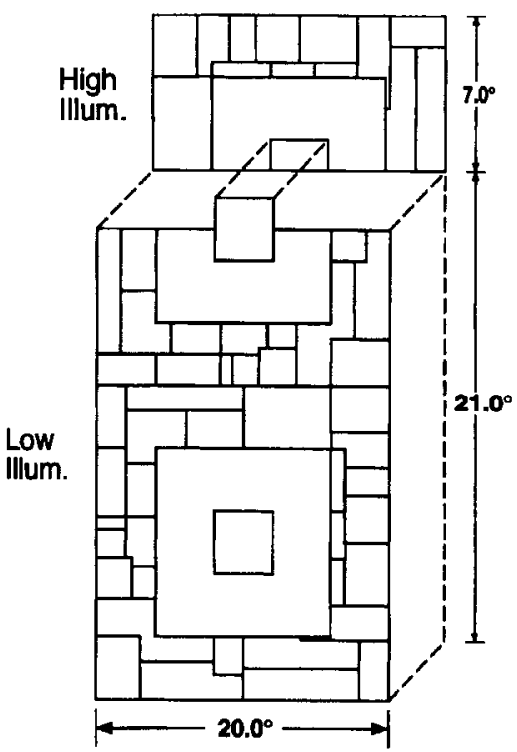

(b)

Figure 1. Diagram of stimulus patterns: (a) entire comparison annulus in near depth plane, under low illumination (1/30 of high illumination); (b) "top half" of the comparison annulus in far depth plane, under high illumination.

unteers (D.A., S.G., I.B., and L.C.) were unaware of the purpose of the experiment.

\section{Procedure}

The observers initially adapted for $3 \mathrm{~min}$ to a $6500 \mathrm{~K}, 68.5$ $\mathrm{cd} / \mathrm{m}^{2}$ uniform white field. The stimulus pattern was then presented continuously. The observers were asked to spend about the same amount of time looking at the test and comparison patches and to alternate their gaze between the two approximately once per second. The observers adjusted the luminance of the test patch to match its lightness to that of the comparison patch. They were instructed to make the test patch "look as if it were cut from the same piece of paper" as was the corresponding comparison patch. Between trials, the computer randomly offset the horizontal position of the luminance mapping within a range of $\pm 10 \%$, to prevent position cues from influencing the matches.

\section{Design}

The observers viewed four different arrays of reflectances. When the comparison patch had a luminance equivalent to Munsell value $V=9.5$ ( (a white), the near plane was dimly illuminated ( $1 / 30$ the illumination of the far plane). Its annular surround (as well as that of the annuli surrounding the lower test patch) could be either Munsell value $\mathrm{V}=3.5$ / (a dark gray) or Munsell value $\mathrm{V}=3.0$ / (a slightly darker gray). When the comparison patch was Munsell value $\mathrm{V}=2.0$ / (a black), the near plane was brightly illuminated ( 30 times the illumination of the far plane). Its annular surround (as well as that of the annuli surrounding the lower test patch) could be either Munsell value $\mathrm{V}=3.5 /$ or $\mathrm{V}=4.0$ / (a slightly lighter gray).

Three runs, each containing five test-patch adjustments presented in pseudorandom order, were made in each of the split- and entire-annulus conditions--with a test Munsell value of $\mathrm{V}=9.5$ / for both $V=3.5 /$ and $V=3.0 /$ annular surrounds, as well as a test Munsell value of $\mathrm{V}=2.0$ / for both $\mathrm{V}=3.5 /$ and $\mathrm{V}=4.0$ / annular surrounds. An experimental session, requiring about $30 \mathrm{~min}$, consisted of presenting the black and white standards in pseudorandom order.

\section{RESULTS}

For comparison with theory, it is convenient to convert the observers' mean luminance settings to Munsell values. Each test-patch luminance was divided by the illumination of the near Mondrian to determine its reflectance and then converted to a Munsell value by using published tables (Newhall, Nickerson, \& Judd, 1943). The average Munsell values as a function of split or entire comparison annuli are shown in Figure 2. Each point is the mean of three runs ( $n=5$ per run) for each of the six observers. The panels on the left are for the $V=3.5$ / annuli and the panels on the right are for the $\mathrm{V}=3.0$ / or $\mathrm{V}=4.0 /$ annuli.

Overall, the pattern of ordinal effects agreed with the prediction: The light comparison patch looked lighter and the dark comparison patch looked darker with the entire-annulus than with the split-annulus. The $\mathrm{V}=9.5 /$ comparison patch was matched in the split-annulus condition by substantially darker grays (approximately $\mathrm{V}=$ 7.5/) than in the entire-annulus condition, in which the matches were closer to veridical. Also, as expected, the $\mathrm{V}=2.0$ / comparison patch was matched by lighter grays (approximately $\mathrm{V}=0.5 /$ Munsell steps) in the split-annulus condition than in the entire-annulus condition.

Within that overall pattern, the mean matches varied from observer to observer and over surround conditions. In the conditions with $\mathrm{V}=3.0 /$ and $\mathrm{V}=4.0$ / annuli, the expected qualitative pattern of matches ( $p<.5$ in all cases) occurred, except on I.B.'s 2.0/ test and D.A.'s 9.5/ test. With the $\mathrm{V}=3.5 /$ annulus, the expected pattern of matches occurred at a statistically significant level $(p<$ .5 in all cases), but the differences were smaller, except 

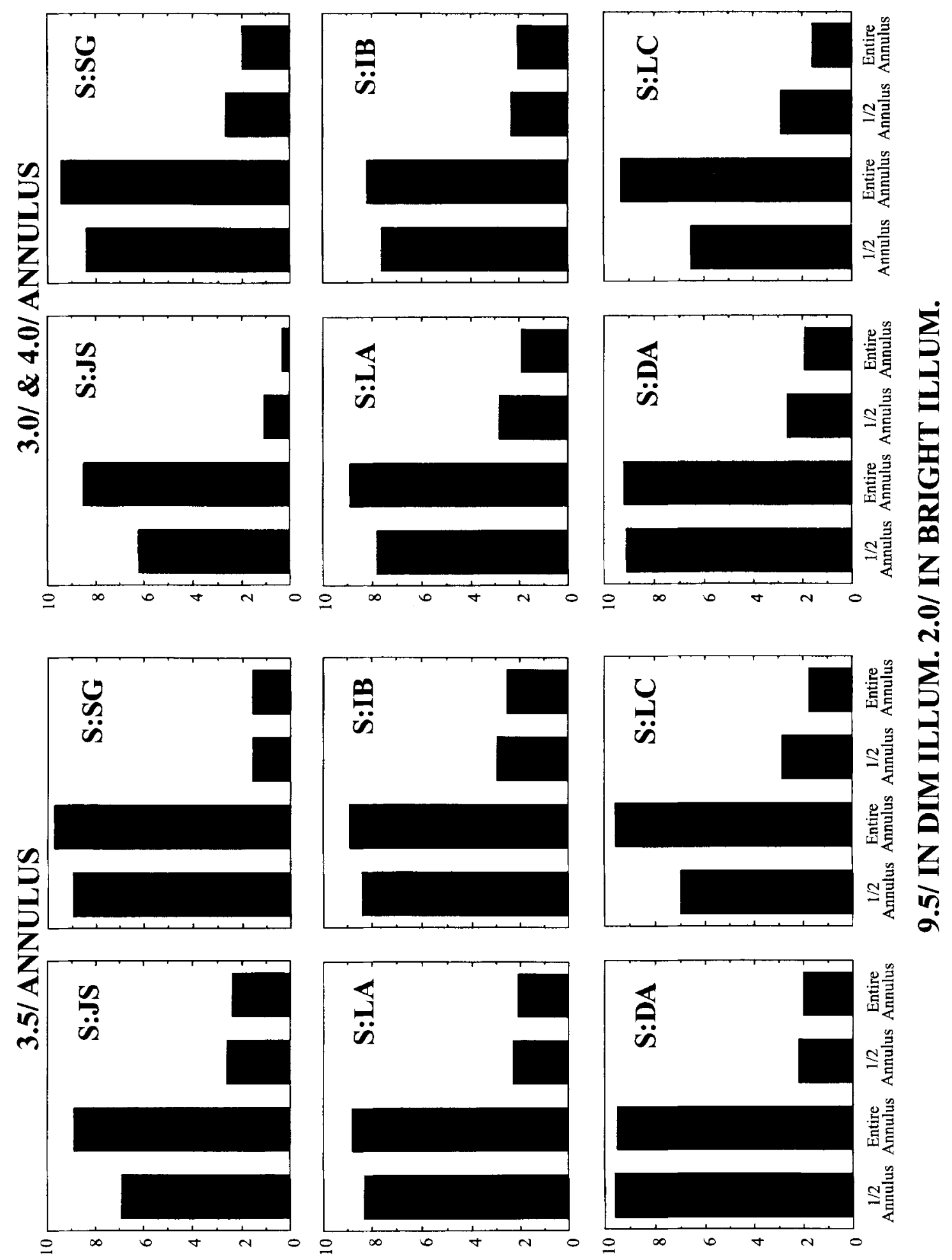

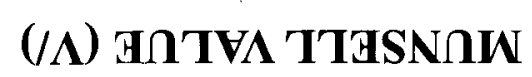

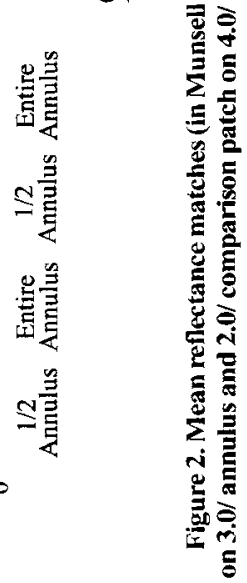


on S.G.'s 2.0/ test and D.A.'s 9.5/ test. Observers L.A., S.G., J.S., and D.A. showed minimal departures $(0.05$ $\log$ units) from constancy for the $V=2.0$ / patch with $V=$ $3.5 /$ annulus.

Figure 3 shows the means over all 6 observers. When the $\mathrm{V}=9.5 /$ comparison and the $\mathrm{V}=2.0 /$ comparison were seen on the $\mathrm{V}=3.5$ / annuli, observers judged the $\mathrm{V}=9.5 /$ comparison as lighter $(p<.02)$ and the $\mathrm{V}=2.0 /$ comparison as darker $(p<.03)$ with the entire annulus than with the split annulus. When the annulus was made darker $(\mathrm{V}=3.0 /)$ for the $\mathrm{V}=9.5 /$ comparison $(p<.02)$, and lighter $(\mathrm{V}=4.0 /)$ for the $\mathrm{V}=2.0 /$ comparison $(p<$ $.01)$, these differences increased.

\section{DISCUSSION}

The data support our hypothesis; the slight departures from lightness constancy found in two earlier studies may be attributed in part to the contrast between a background field and a retinally adjacent test field perceived to lie in different depth planes. In Gilchrist (1977) and Schirillo et al. (1990), a white patch in a brightly illumi-
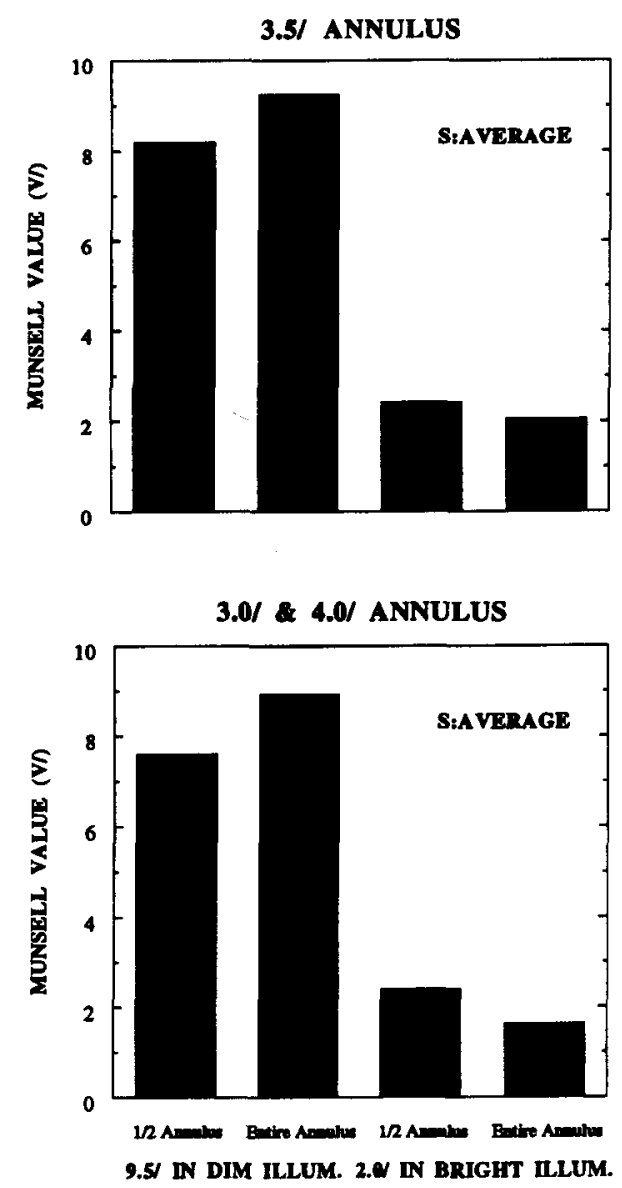

Figure 3. Mean reflectance matches (in Munsell value units), average of 6 observers. Top: 9.5/ and the 2.0/ comparison patches on 3.5/ annulus. Bottom: 9.5/ comparison patch on 3.0/ annulus and 2.0/ comparison patch on 4.0 / annulus. nated far room and a black patch in a dimly illuminated near room were always visible. Each was retinally adjacent to a test patch independently of the room in which the test appeared to lie. The present data indicate that slight departures from lightness constancy occurred when the comparison patch was immediately adjacent to the background field, whereas lightness constancy was nearly perfect when a nearly $3^{\circ}$ gray buffer field separated the comparison from the background. ${ }^{4}$

A number of authors have argued that neural interactions involving contrast are stronger between coplanar fields than between fields in different depth planes (Gilchrist, 1977; Wist, 1974; Wist \& Susen, 1973). However, we showed in our previous paper (Schirillo et al., 1990) that the brightness of the test patch was unaffected by depth changes that dramatically altered its coplanar surround. Our clear distinction between lightness and brightness matches (see note 1) revealed that Gilchrist's data should be understood as a lightness difference due to the apparent illumination change when the apparent depth changed, rather than a brightness contrast effect.

Koffka (1932) reproduced, extended, and analyzed the Gelb effect. His analysis illustrates the close connection between that effect and Gilchrist's (1977) and Schirillo et al.'s (1990) experiments. Koffka argued that the shift in appearance of the target surface from white to black was a consequence of its becoming closely organized with the small white target. In one of his extensions of Gelb's experiments, the change of scene organization involved simultaneous changes of apparent depth and lightness. In Gilchrist's and Schirillo et al.'s experiments, apparent depth was used in order to establish a relationship between the target and the far room patches, with their high illumination, or the near room patches, with their dim illumination. It seems likely, therefore, that the arguments presented above apply to the Gelb effect as well as the Gilchrist paradigm. The change of scene organization involves large changes of the target's lightness, but its brightness should be relatively unaffected. ${ }^{5}$ It goes from appearing to be a white surface under dim illumination to appearing to be a black surface under high illumination, but the resulting brightness is relatively constant.

The current data support the conjecture that the departures from perfect lightness constancy in Gilchrist's (1977) and Schirillo et al.'s (1990) experiments were in part due to local contrast effects among regions that were retinally adjacent but separated in depth. With the $3.0 /$ and $4.0 /$ annuli, the local contrast effects constricted the lightness scale of the comparison patch by 2.0 Munsell steps, whereas the $3.5 /$ annulus restricted it by 1.4 Munsell steps. Gilchrist's matches ranged from 3.5/ to 9.0\% Adding 2 Munsell steps to that range accounts for the $30: 1$ ratio predicted by perfect lightness constancy. ${ }^{6}$

Cataliotti and Gilchrist (in press) have suggested that the effects we observed are due not directly to local contrast change, but to the illumination change per se. They propose that the highest luminance under a single illumination creates an anchoring compromise, and that sur- 
faces along the border of two illuminations are most affected. In our experiment, the change in local contrast was the result of an illumination change, so a separate series of experiments will be required in order to uncouple these two factors.

It is also noteworthy that Schirillo et al.'s (1990) display exhibited a smaller degree of constancy than did its related split-annulus condition in the current work. This "may be due to the minimal number of patches in the front room. Although these two patches span the luminance range of physical reflectances, they may not be sufficient to define a perceptual gray scale adequately" (Schirillo et al., 1990, pp. 88-89). Additional work by Cataliotti and Gilchrist (1994) suggests that an increase in articulation, or the number of surface reflectances under a given illumination, might have expanded Schirillo et al.'s (1990) perceived white/black range. Additional research to explore the effects of both an anchoring compromise and articulation would characterize reasons for departures of constancy that exist in the literature.

\section{REFERENCES}

AREND, L. E., \& AREND, D. M. (1994). Both lightness and brightness are affected by background reflectance. Manuscript in preparation. AREND, L. E., \& GoldsteIN, R. (1987a). Lightness models, gradient illusions, and curl. Perception \& Psychophysics, 42, 65-80.

AREND, L. [E.], \& Goldstein, R. (1987b). Simultaneous constancy, lightness and brightness. Journal of the Optical Society of America $A, 4,2281-2285$.

AREND, L. [E.], \& Goldstein, R. (1990). Lightness and brightness over spatial illumination gradients. Journal of the Optical Society of America A, 7, 1929-1936.

AREND, L. [E.], \& ReEves; A. (1986). Simultaneous color constancy. Journal of the Optical Society of America A, 3, 1743-1751.

AREND, L. E., \& SPEHAR, B. (1993). Lightness, brightness, and brightness contrast: 1. Illuminance variation. Perception \& Psychophysics, 54, 446-456.

Cataliotti, J., \& Gilchrist, A. L. (1994). Anchoring of surface lightness with multiple illumination levels. Investigative Ophthalmology \& Visual Science, 35, 2165.

Cataliotti, J., \& Gilchrist, A. [L.] (in press). Local and global processes in surface lightness perception. Perception \& Psychophysics.

EASTMAN KODAK COMPANY (1986). Kodak color films and papers for professionals. Rochester, NY: Author.

Gilchrist, A. L. (1977). Perceived lightness depends on perceived spatial arrangement. Science, 195, 185-187.

GILCHRIST, A. L. (1980). When does perceived lightness depend on perceived spatial arrangement? Perception \& Psychophysics, 28, 527-538.

Gilchrist, A. [L.], Delman, S., \& Jacobsen, A. (1983). The classification and integration of edges as critical to the perception of reflectance and illumination. Perception \& Psychophysics, 33, 425436.

Heinemann, E. G. (1955). Simultaneous brightness induction as a function of inducing- and test-field luminances. Journal of Experimental Psychology, 50, 89-96.

JACOBSEN, A., \& GILCHRIST, A. [L.] (1988). The ratio principle holds over a million-to-one range of illumination. Perception \& Psychophysics, 43, 1-6.

KOFFKA, K. (1932). A new theory of brightness-constancy: A contribution to a general theory of vision. Report of a Joint Discussion on Vision by the Physical and Optical Societies. Cambridge: The Physical Society.
LAND, E. H., \& MCCANN, J. J. (1971). Lightness and retinex theory. Journal of the Optical Society of America, 61, 1-11.

Leibowitz, H., Mote, F. A., \& Thurlow, W. R. (1953). Simultaneous contrast as a function of separation between test and inducing fields. Journal of Experimental Psychologv, 46, 453-456.

MCCANN, J. (1987, June). How is color constancy to be explained? Paper presented at the International Color Association Interim Meeting, Florence.

Mingolla, E., \& AREnd, L. E. (1993, May). Lightness constancy in $3 D$ scenes. Paper presented at the International Conference on Event Perception and Action, Vancouver.

Newhall, S. M., Nickerson, D., \& Judd, D. B. (1943). Final report of the OSA subcommittee on spacing of the Munsell colors. Journal of the Optical Society of America, 33, 385-418.

Schirillo, J., Reeves, A., \& Arend, L. [E.] (1990). Perceived lightness, but not brightness, of achromatic surfaces depends on perceived depth information. Perception \& Psychophysics, 48, 82-90.

Todd, J. T., \& Mingolla, E. (1983). Perception of surface curvature and direction of illumination from patterns of shading. Journal of Experimental Psychology: Human Perception \& Performance, 10, 734-739.

WhitTLE, P. (1992). Brightness, discriminability and the "crispening effect." Vision Research, 32, 1493-1507.

WIST, E. R. (1974). Mach bands and depth adjacency. Bulletin of the Psychonomic Society, 3, 97-99.

WIST, E. R., \& SUSEN, P. (1973). Evidence for the role of post-retinal processes in simultaneous contrast. Psychologische Forschung, 36, 1-12.

\section{NOTES}

1. Lightness refers to apparent reflectance, ranging from blacks through grays to whites. Brightness refers to perceived luminance, the overall effective intensity of the stimuli or stimulus.

2. Further details of apparatus, linearization, and calibration are given in Arend and Reeves (1986) and Arend and Goldstein (1987b)

3 . The $1280 \times 1024$ pixel screen was set to provide a steady, neutral background with an average luminance of $17.4 \mathrm{~cd} / \mathrm{m}^{2}$, and CIE chromaticity $x=0.33$ and $y=0.33$. The scan rate was $60 \mathrm{~Hz}$, noninterlaced. The red, green, and blue guns were linearized by using a 9-bit look-up table. A given chromaticity and luminance, set by software, did not vary appreciably over the effective viewing area. The luminance was approximately constant $( \pm 3 \%)$ within the central region of the screen that displayed the Mondrians.

4. In the near depth condition, the comparison patch differed from the test only in its distance from the illumination border. The $2.75^{\circ} \mathrm{sep}-$ aration from the comparison patch edge to the illumination edge should have been sufficient to minimize local contrast (see, e.g., Leibowitz, Mote, \& Thurlow, 1953), but it may still have produced the residual departure from exact lightness constancy. For example, Observers J.S., L.A., and I.B. judged the $9.5 /$ comparison patch to be less than $9.0 /$ and the $2.0 /$ comparison to be slightly greater that $2.0 \%$.

5. Unlike the Gilchrist (1977) and Schirillo et al. (1990) designs, the Gelb paradigm involves introduction of an additional patch of high luminance. Some change of brightness due to local contrast effects is thus expected, but the change should be small relative to the lightness change. A direct Gelb effect experiment with properly defined brightness and lightness tasks is needed.

6. This might also account for Condition 8 of Gilchrist's (1980, part 2) tab experiment. Bending the tabs over so that they lie flat on their retinally adjacent surrounds is analogous to our placing the upper half of the annulus in the near depth plane. This would allow any perceived differences in the edge to be attributed to differences in reflectance and not be confounded with differences in illumination. This should restore the expected 5.5/ Munsell difference from 1.25/ by completing the coplanarity.

(Manuscript received March 7, 1994; revision accepted for publication September 29, 1994.) 